\title{
Effect of the Combination of Biofeedback and Sequential Psychotherapy on the Cognitive Function of Trauma Patients Based on the Fusion of Set Theory Model
}

\author{
Y. ZHANG AND Q. YUAN* \\ Tangshan Workers' Hospital, Tangshan, Hebei Province, 063000 China
}

Zhang and Yuan: Effect of the Combination of Biofeedback and Sequential Psychotherapy on the Cognitive Function of Trauma Patients Based on the Fusion of Set Theory Model

\begin{abstract}
This study intended to take a special group of trauma patients as research subjects to propose a method analysing the effect of combination of biofeedback and sequential psychotherapy based on the fusion of the set theory model on the cognitive function of these patients with trauma. The occurrence and development of post-traumatic stress disorder and the cognitive function is investigated. The set theory model is used in this study to carry out a survey on the effect of the combination of biofeedback and sequential psychotherapy on patients with post-traumatic stress disorder to describe the occurrence, development, change trajectory and time course characteristics of post-traumatic stress disorder. The set theory model was employed to investigate the cognitive development characteristics of these trauma patients. In addition, through the set theory model, psychological behavior mechanism for the occurrence and development of post-traumatic stress disorder is revealed. The study of the combination of biofeedback and sequential psychotherapy is adopted to investigate the effect of the post-traumatic stress disorder on the cognitive function of the trauma patients. The results of this study could be used to provide scientific advice for the placement and psychological assistance of trauma patients in future, to provide a scientific basis for a targeted psychological intervention and overall planning of the intervention, and to provide scientific and objective indicators and methods for the diagnosis and assessment of intervention of traumatic psychology in patients with trauma in the future.
\end{abstract}

Key words: Markov model, severe heart failure, treatment and nursing care, coronary artery bypass graftingp

Obsessive-Compulsive disorder (OCD) refers to the recurring and confusing concept and/or the repetitive and irrepressible behavior, accompanied by an emotional experience of anxiety and pain, which has influenced the learning and work of the patients or even led to negative impacts on their daily life[1,2]. OCD is the fourth most common mental illness after depression, alcohol dependence and phobia. The average age of onset is 22 to $36 \mathrm{y}$. The incidence rate is similar between male and female. It is prone to occur in special populations, such as the unemployed and the group with relatively poor economic situation, the divorced and single population, the group with single-parent family and so on[3,4]. It has been reported that the patients suffering from OCD can be severely impaired in their ability to read and maintain attention, which suggests that there are serious defects in the cognitive function of the patients with OCD[5]. The sudden, unpredictable traumas that are severely destructive with major casualties not only can cause serious psychological stress to the family members and relatives of the trauma patients, but also can bring about negative impacts[6,7]. For example, the occurrence of sudden and devastating trauma, the huge property loss and casualties thus caused, the terrible and difficult scenes that the survivors have experienced, the possible injury, the possible loss of loved ones and the further possible psychological trauma thus suffered[8]. In view of the entire psychological study after the traumatic events, many of them have shown that traumatic events such as experiences through difficult situations have a negative impact on the psychology and behavior of people, for example, the occurrence of cognitive bias, the generation of psychological stress, depression, 
anxiety or even severe post-traumatic stress disorder (PTSD)[9-10].

For trauma patients and adolescents who have experienced traumatic events, PTSD, anxiety and depression are the 3 most common clinical problems and there is also the possibility of comorbidity. This is because the trauma patients lack ability of cognition and self-regulation. They have relatively low psychological defense ability, strong susceptibility to trauma and often have more severe psychological reactions to the sudden and destructive difficult events. Hence, they are more prone to have cognitive reactions and thus become a group with high risk of the incidence of PTSD and become subjects for combination of biofeedback and sequential psychotherapy. The trauma patients and adolescents of different ages have different psychological and behavioral responses after they experience trauma. The symptoms of the trauma patients who undergo PTSD are different from those of the adults. Hence, there are differences in their clinical manifestations and diagnosis[11]. The traumatic re-experience symptoms in trauma patients can be expressed as nightmares, repeated play of the traumatic events, emotional excitement or sadness when faced with relevant prompts when playing the theme game related to trauma, and so on. Avoidance symptoms often appear in trauma patients, which are manifested as dissociative anxiety, being clingy and unwilling to leave the parents; high alert symptoms are often shown in the trauma patients as excessive startle response, high vigilance, attention disorder, irritability or rage, difficulty to fall asleep and so on. In addition, the manifestations of PTSD in the trauma patients of different age group may also be different.

The differences in the response of trauma patients and adolescents with different types of trauma (such as those whose classrooms have collapsed but who have not been injured, those who have witnessed injuries or deaths of their teachers or classmates, those who faced death of their parents or family members and those who suffer from injuries and disabilities) are even greater. For those trauma patients who have lost their parents due to trauma and thus become trauma patients themselves not only suffer from the horror of the traumatic scene to endure the great shocks, but also suffer from the great grief of losing their parents and may even have to lead a life drifting from place to place ever since. The double injuries will undoubtedly have a greater impact on their psychology. Studies on patients of traumatology have shown that the trauma patients have relatively many psychological problems after they suffer from psychological trauma, which are manifested as psychological and behavioral problems such as anxiety, depression, somatization, hostility, fear, fatigue, anger and so on, the incidence of which is significantly higher than that in the nontraumatic patients who have faced similar traumatic experiences[12,13]. The incidence of the PTSD in these trauma patients is still as high as $23 \%$. From the studies reported previously, it is found that the probability of patients treated with a combination of the biofeedback and sequential psychotherapy is higher under PTSD than the non-trauma patients treated with a combination of biofeedback and sequential psychotherapy (33.93 and $22.22 \%$ ), the difference is significant, and the trauma patients treated with the combination of biofeedback and sequential psychotherapy may often experience the trauma again or have the avoidance behavior. The individuals who have higher degree of psychological cognitive response to the combination of biofeedback and sequential psychotherapy often have poorer selfawareness and the probability score of the PTSD is significantly correlated with the self-awareness scale. Similarly, for the selected samples, the positive rate of the PTSD detection did not decrease significantly after $1 \mathrm{y}$. This indicated that the impact of PTSD would persist for a long time. In addition, some of the subjects turned from PTSD negative to positive after $1 \mathrm{y}$, which suggested individual differences in the lag period of PTSD, which required a follow-up study to explore the changes in the PTSD[14].

The above studies have shown that trauma patients have relatively many psychological problems and constitute a high risk group for PTSD. Therefore, it is necessary to screen patients with trauma after the traumatic events in a timely manner and carry out psychological intervention to those individuals who are prone to develop PTSD, so as to minimize psychological damage caused by trauma. PTSD is a kind of mental disorder caused by a variety of factors, which is initially initiated by high intensity cognitive sources, and then maintained by a number of cognitive, internal and external factors. Therefore, the measurement of PTSD in trauma patients and adolescents must take the development and the social situation factors into consideration. Compared to other trauma patients, the family function and attachment relationship of PTSD patients are impaired and the development of PTSD has its own characteristics. However, literature currently available revealed that there were very few studies that specifically investigated the psychological behaviors of 
trauma patients both in china and abroad. This suggested the immediate necessity to explore the characteristics of psychological and behavioral development in patients with trauma. It essential to understand the psychological behavior characteristics of patients with different trauma levels and different cultural backgrounds at different time points in the combination with the biofeedback and sequential psychotherapy. In this way, it is possible to provide a strong theoretical basis to develop the macro decisions along with scientific basis for employing psychological assistance and evaluating its effectiveness. Therefore, it is necessary to screen and diagnose PTSD in patients with trauma and use the set theory model to carry out tracking investigation on PTSD in trauma patients in different trauma areas, who are treated with the combination of biofeedback and sequential psychotherapy for a long term. This would not only provide timely guidance for mental health promotion, but also examine the effectiveness of these interventions and the characteristics of PTSD patients with different lag periods, thus revealing the development and progression of the PTSD in different individuals at different stages after trauma[15].

The application of the combination of biofeedback and sequential psychotherapy in the treatment of trauma often showed different symptoms and cognitive functions from other mental illnesses and it will significantly affect the psychological and neuronal immune system of the body. In the studies based on adults as test subjects, it was found that the PTSD patients presented a number of cognitive deficits, including relatively slow cognitive processing speed, relatively poor reasoning ability (such as the attention defect, memory impairment, impaired executive function). Animal studies on the standardized neuropsychology have shown that in terms of acute or chronic cognitive dysfunction, high-dose cortisone can cause hippocampal neuron atrophy with memory defects. In fact, many of the symptoms of PTSD are directly or indirectly related to the attention, memory and other cognitive functions. For example, attention deficit is one of the diagnostic criteria for PTSD high alert symptoms and the difficulty of inhibition in the executive function is a feature of the PTSD intrusion dimension. The degree of trauma is linearly related to the probability of developing PTSD symptoms. Trauma patients who suffered double major trauma personally have their PTSD symptoms significantly higher than those of the normal trauma patients, which may further impair their cognitive function. At the same time, as developing trauma patients, their cognitive functions such as the attention and the memory are constantly developing as well. Hence, the extent of damage to cognitive function of PTSD patients after they experienced double major trauma and the neural mechanisms behind this damage have not been investigated either in China or abroad. With the continuous advancement in the research methods and techniques, it became possible to explore deeply to develop a greater understanding. For example, the eye movement tracking technology has the unique advantage of recording the time and the spatial information in real time. The technical method of the cognitive function science can be used to investigate the neurological mechanism problem that is hidden behind the data. In summary, it is necessary and urgent to carry out scientific research on the development and the mechanism of PTSD in the trauma patients.

\section{MATERIALS AND METHODS}

In this investigation, the set theory model is adopted in the analysis on the effect of the combination of biofeedback and sequential psychotherapy on the cognitive function of trauma patients. Therefore, it is necessary to introduce the relevant knowledge of the set theory model briefly in this paper. The single part of the cardinal number $\mathrm{M}$ in the $\mathrm{m} \times \mathrm{n}$ can be decomposed and multiplied as the following, $\mathrm{m} \times \mathrm{m}$ and $\mathrm{W}, \mathrm{m} \times \mathrm{n}$ and $\Sigma, \mathrm{n} \times \mathrm{n}$ and $\mathrm{V}$. The details are as the following, $\mathrm{Mm} \times \mathrm{n}=$ $\mathrm{Wm} \times \mathrm{m} \times \Sigma \mathrm{m} \times \mathrm{n} \times \mathrm{VTn} \times \mathrm{n}$, in which, $\Sigma=\left[\begin{array}{ccc}\sigma_{1} & 0 & 0 \\ 0 & \sigma_{2} & 0 \\ . . & . . & . .\end{array}\right]$ (1) indicates that for the singular value matrix, its diagonal elements are $\sigma 1, \sigma 2, \ldots$, which is referred to as the singular value of the matrix. Its property is nonnegative decreasing and all the other elements are 0 . $\mathrm{W}=(\mathrm{w} 1, \mathrm{w} 2, \ldots, \mathrm{wi}, \ldots, \mathrm{wm})$, in which wi stands for the left vector related to $M$, for the basic form of the response $\mathrm{M}$, larger the corresponding ratio oi indicates that the property of the characteristic ui of $\mathrm{M}$ is larger; in fact, ui is the eigen vector of the square matrix MMT.

$\mathrm{V}=(\mathrm{v} 1, \mathrm{v} 2, \ldots, \mathrm{vi}, \ldots, \mathrm{vn})$, in which vi stands for the right vector related to $M$. In addition, the characteristics of $\mathrm{M}$ is described, which is corresponding to $\sigma \mathrm{i}$; at the same time, if the value of $\sigma \mathrm{i}$ is larger, the property of the characteristic vi will also be increased accordingly; in fact, vi is the comparison vector of the square matrix MTM as the following

$\hat{\Sigma}=\left[\begin{array}{ccc}\sigma_{1} & 0 & 0 \\ 0 & \sigma_{2} & 0 \\ . . & . . & \sigma_{r}\end{array}\right], \hat{W}=\left(u_{1}, u_{2}, \ldots, u_{r}\right), V=\left(v_{1}, v_{2}, \ldots, v_{r}\right)$ 
Hence, it can be found that the approximate matrix of the original matrix is as the following.

$$
M_{m \times n} \approx \hat{M}_{m \times n}=W_{m \times r} \times \hat{\Sigma}_{r \times r} \times V_{r \times n}^{T}
$$

The basic idea is introduced as the following, the main features of the cognitive function effectiveness evaluation matrix of the trauma patients are extracted by using the set theory model, and then these characteristics are taken as the basic objects to calculate the degree of similarity between the trauma patients. In addition, the content of trauma patients where the cognitive function evaluation has not been carried out is filled accordingly.

\section{RESULTS AND DISCUSSION}

This research focused on the systematic study of the scientific issues related to the occurrence, development and cognitive mechanisms of trauma patients under PTSD after significant trauma. The problem can be broken down into the following aspects, through the integration of the theoretical model with the combination of the biofeedback and the sequential psychotherapy, the trajectory and the time course characteristics of the occurrence, development and change of the psychological trauma in trauma patients are described. The psychology of the patients with trauma under PTSD treated with the combination of biofeedback and sequential psychotherapy is investigated. The comparative study of the set theory model is carried out to investigate the characteristics of the psychological cognition development in trauma patients. In addition, the cognitive mechanism of the occurrence and development of the psychological trauma in trauma patients is revealed through the set theory model. The eye movement tracking technology and the EEG technology were used to carry out in-depth exploration for the effect of PTSD on the cognitive function in trauma patients and the comparative study is conducted to investigate the effect of PTSD on the cognitive function of trauma patients.

From the existing studies of the combination of combination of biofeedback and sequential psychotherapy, it can be found that the degree of traumatic damage to the patients who are located in the traumatic event is significantly higher than those in the other cities, as their objective and subjective experience are more severe than those in the other cities. Therefore, in this study, the following aspects were investigated. The rate of post-traumatic stress disorder in trauma patients with different levels of trauma, different ages and different genders at different time points in the combination of biofeedback and sequential psychotherapy. The relationship between the traumatic cognitive dysfunction and other mental health issues such as anxiety, depression and so on.

In order to describe the occurrence, development, change trajectory and time course characteristics of the trauma patients, a method that integrated the set theory model with the combination of the biofeedback and the sequential psychotherapy is adopted in this study. The overall research design roadmap is shown in fig. 1.

At the same time, in accordance with the previous related studies, 5 questions designed to investigate the traumatic experience of trauma patients were developed. Whether the patient was trapped, injured, witnessed death of a loved one or the death of another person, or suffered from the death of a loved one, such descriptions would serve as independent variables to carry out the regression analysis. On the basis of the results of the post-traumatic cognitive response studies in trauma patients treated with the combination of biofeedback and sequential psychotherapy, it is expected that PTSD in trauma patients should be affected by the degree of trauma, the experience of trauma and the years of age. The probability of trauma patients under PTSD is much higher than the normal trauma patients; the probability in the trauma patients under PTSD in mild trauma area is less than the trauma patient in the severe trauma area; the probability of the trauma patients under PTSD is

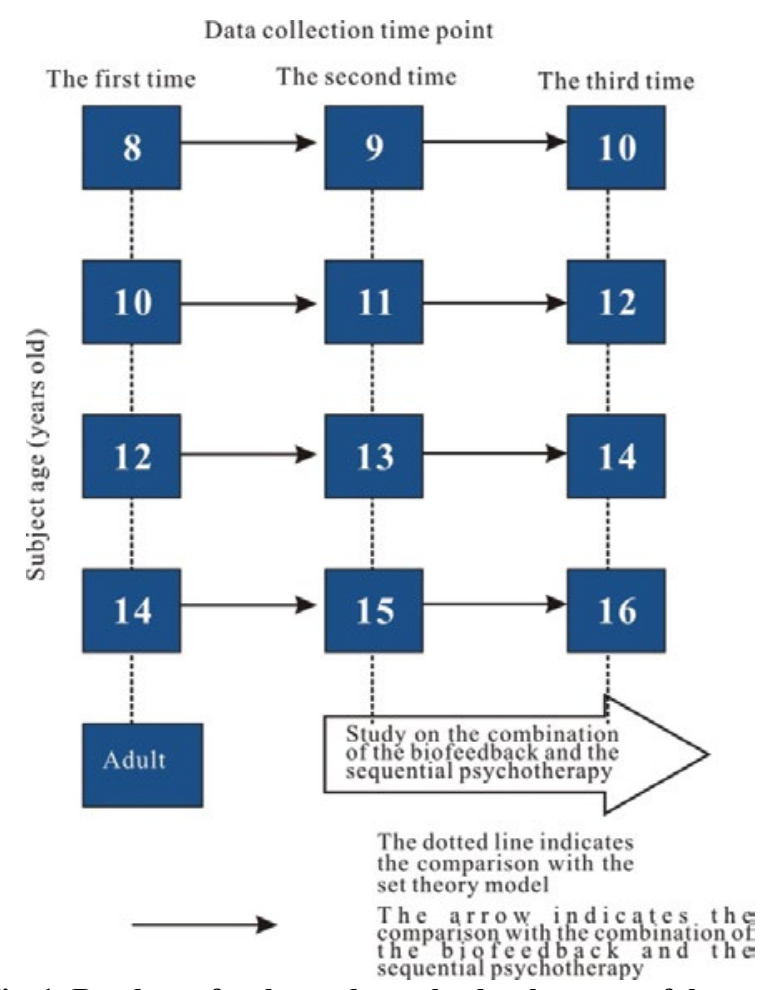

Fig. 1: Roadmap for the study on the development of the trauma patients 
closely related to their age and the PTSD symptoms are nonlinearly changed with increasing age.

The follow-up survey on the trauma patients in Sichuan after the trauma is carried out by using a revised version of the Trauma Patient Event Impact Scale to investigate the occurrence and development characteristics of the PTSD. The test subject samples investigated at the end of 2008 are tracked. The time points for the collection of the data were May 2011, May 2012, May 2013 and May 2014 and the tracking research is carried out accordingly. In the survey, classes are adopted as a group to carry out the group testing. The psychologists served as the experimenters. In addition, the survey methods and instructions are unified for the testing. The data obtained at the end of 2008 and 2009 were combined and comparison analysis was carried out. The PTSD rate is measured by the chi-square test, and variance analysis is carried out on the PTSD symptoms; in addition, in-depth analysis on the data of the conversion probability from high to low in the PTSD in the process of tracking (turning negative and turning positive) is carried out, which could provide a basis for the exploration of the occurrence and development mechanism of PTSD. In addition, it is also expected to provide a theoretical basis for the evaluation of the psychological assistance in the future.

The cognitive function of the trauma patients has a very close relationship with their academic and career achievements in future. Through the investigation of cognitive function in the combination of biofeedback and sequential psychotherapy, the problems could be identified in a timely manner and it would be conducive to developing better cognitive functions in a more effective manner, so that the patients can better exert their potential in their studies and their work in future. This study has shown that the difficulty that occured in the attention and memory is a key feature of PTSD. In this study, the characteristics of the attention, memory, intelligence and creativity in the trauma patients with different ages and genders treated with the combination of biofeedback and sequential psychotherapy were investigated.

The set theory model was used to carry out a questionnaire survey and the laboratory cognitive experiments to investigate the developmental characteristics of the cognitive function in patients with trauma at different age groups and their relationship with PTSD. Prior to the investigation, the guardian and the adoption institution of the trauma patients or the responsible leader of the school would sign a written informed consent. Cognitive functions included the following, Raven's standard intelligence test (intelligence), "A $\rightarrow \mathrm{X}$ " continuous working tasks (retentive attention) and visual search tasks (selective attention), working memory tests (memory refresh, attention conversion and the inhibition of prepotent responses). Procedures for the testing included the Raven's Standard Progressive Matrices Test for the cognition, the group testing is adopted; and for the other cognitive tasks, the computerized (for the stimulus presentation and response recording) individual testing is carried out. The PTSD data collected in the same year were combined. In addition, the multivariate analysis of variance and the set theory model were used to explore the relationship with the PTSD. In accordance to previous studies and the existing research on PTSD of trauma patients treated with the combination of biofeedback and sequential psychotherapy, it was expected that the attention maintenance and selective attention, memory breadth and memory refresh can significantly predict the PTSD symptoms in the reverse direction; while the false alert rate of the attention (which is used to reflect the ability to inhibit the non-targets) can significantly predict PTSD symptoms in the forward direction. Characteristics of the PTSD and non-PTSD on the Eye Movement Tracking of the Cognitive Function in the Patients with Trauma Eye movement tracking recording technology referred to a kind of research method where the eye movement tracker is used to record and analyze various eye movement tracking indicators when people are reading, browsing pictures, viewing the outdoor scenes and carrying out the visual cognitive tasks, so as to reveal the process and the pattern of the psychological processing in people. The eye movement tracking can reflect the information processing process of the human brain. The characteristics of the eye movement tracking pattern are closely related to the information processing activities of the brain. Hence, the eye movement tracking research is the most effective instant research method in the study of the dynamic cognitive processes. The use of the eye tracking technology has made it possible for the progress of the scientific research of many visual cognitive tasks with a big step forward: It can record the time, position and sequence of the individuals in processing the visual stimuli in an accurate, objective and detailed manner in real time, and truthfully reproduce the processing behavior of the individuals. This is a kind of method that combines the qualitative and the quantitative analysis.

As a kind of non-invasive technique, the eye movement tracking technology has been widely used to investigate 
the cognitive processes and the neural basis of the normal and abnormal populations. The eye movement tracking technology has been applied in foreign countries to investigate cognitive functional basis of individuals who suffer from mental disorders, such as autism, schizophrenia, dyslexia, anxiety, depression and so on. Up until now, this technology has provided researchers with more possibilities to research on the social and emotional development. For example, in the study of individuals as test subjects, the most consistent result obtained by using the eye movement tracking technology is that individuals presented relatively early saccade. In addition, relatively more errors occur in the antisaccade tasks, which reflected the difficulty of suppressing function and this is also a symptom of patients with PTSD. At the same time, depressed adolescents showed relatively poor antisaccade latency in the antisaccade tasks than that in healthy adolescents. This indicated that the reaction inhibition rate is relatively poor. Therefore, it could be concluded that the eye movement tracking technology could also be applied to the studies of cognitive function in patients with PTSD. In this study, the eye movement tracking characteristics of trauma patients with and without PTSD were investigated in the attention and memory tasks.

In accordance with the results of previous investigations, trauma patients with and without PTSD treated with the combination of biofeedback and sequential psychotherapy were selected to investigate eye movement characteristics during the attention and memory tasks. Trauma patients with family psychiatric history and those patients whose visual acuity or corrected visual acuity failed to reach normal levels were eliminated. Prior to the experiment, the guardian and the adoption institution of the trauma patients or the responsible leader of the school are required to sign a written informed consent form. Participants in the study group served as experimenters to carry out individual testing under a quiet environment. The Eyelink II eye view monitoring system was used to collect the data. Antisaccade tasks, visual search tasks, 0-back and 1-back working memory tasks were employed. The latency for the initiation of saccade, the accuracy, the fixation time, the pupil diameter, the variation in response, as well as the gaze trajectory in the qualitative description visual search task were all monitored and the data was analysed using multivariate variance analysis.

In terms of the cognitive function, there are also some research results that can be drawn on. As early as 2004, the researchers in China carried out a series of cognitive and functional science studies on 58 military patients and 52 healthy soldiers and found that there was a certain correlation between the PTSD and the auditoryevoked potential, visual-evoked potential, brainstemevoked potential and P300. These indicators could be used as the diagnostic and therapeutic indicators for PTSD to a certain extent. After the May 12 Wenchuan earthquake trauma, West China Hospital of Sichuan University carried out resting state scanning on 44 PTSD patients treated with a combination of biofeedback and sequential psychotherapy for 25 days. The results showed that, compared with the control group, the anterior limb system and striatum activity in the PTSD patients were significantly enhanced. In addition, the connection between the limbic system and the striatum was weakened, which indicated that the brain function of the survivors of the Wenchuan earthquake trauma had changed. The Institute of psychology, Chinese Academy of Sciences also carried out ERP study on 27 survivors of the Wenchuan earthquake trauma. The results showed that their sensitivity of the attention to things was also increased (the MMN volatility amplitude was larger). In addition, they showed more expectation and anxiety for the upcoming stimulus (the CNV volatility amplitude was larger). This corresponded to the high alertness of the PTSD. For example, they may make a very huge response when hearing a very small noise, and they may be startled and so on. All the above studies have shown that PTSD has a certain physiological basis in the human brain. Whether it is the brain structure or the abnormal changes of brain waves, it has provided a basis for the cognitive function science in the diagnosis and treatment of various symptoms of the PTSD. In this study, the changes of the EEG in trauma patients during the process of the attention and memory tasks were also investigated.

In accordance with the results of previous investigations, trauma patients with and without PTSD treated with the combination of biofeedback and sequential psychotherapy were selected to investigate ERP characteristics in the 2 groups in the attention and memory tasks. Trauma patients with the family psychiatric history and the trauma patients whose visual acuity or corrected visual acuity failed to reach the normal levels were eliminated. Prior to the experiment, the guardian and the adoption institution of the trauma patients or the responsible leader of the school are required to sign a written informed consent form.

The research team served as the experimenters to carry out individual testing under a quiet environment. The 
acquisition of the cognitive function signals is carried out using the Neuroscan electroencephalograph, SynAmps2 amplifier, recording with the 64 leads, online filtering $0.05-100 \mathrm{~Hz}$, sampling rate of $500 \mathrm{~Hz}$, and the tip of the nose is taken as the reference point.

Visual search task, go/no-go task, 0-back and 1-back working memory task were investigated. P1, N1, N2 and P3 were used as indictors and the data collected was analysed using multivariate variance analysis. This study aimed at the international frontier in the topic selection and takes the special group of trauma patients as the research subject to investigate the problem of cognitive function in the occurrence and development of PTSD. In the design, the set theory model was integrated with the combination of biofeedback and sequential psychotherapy, which not only can be used to understand the characteristics and the overall trend of the traumatic response and the psychological behavior development in the patients with trauma, but also can reveal the factors that could influence the traumatic response of trauma patients in a relatively objective manner. In the selection of the key technologies in this study, the eye movement tracking and the EEG technology were used to carry out indepth investigation on the cognitive function of PTSD. In particular, on the basis of the analysis of the existing literature, bold inference is made and the eye movement tracking technology is applied to the investigation of understanding the occurrence and the development of the cognitive mechanism of trauma patients under PTSD, which is done for the first time either in China or elsewhere. Eye movement tracking could be used to investigate the spatial characteristics of trauma patients in completing the cognitive tasks very well, and the EEG study could examine the characteristics of the time course very well. The combination of both the eye movement tracking and the EEG study can achieve mutual verification and supplementation.

The investigation, on one hand, allowed the research on the psychological behavior of trauma patients in China to achieve the international frontier level in this field and on the other hand, it could also provide the basis for more targeted national decision-making and the establishment of the combination of biofeedback and sequential psychotherapy assistance program for the treatment of the trauma patients and the implementation of the assistance program in China. In this study, through the design that combined the set theory model research with the combination of biofeedback and sequential psychotherapy, the behavior, cognition and neural mechanism of the traumatic response in trauma patients were explored systematically to obtain the occurrence, development and change trend diagram of trauma patients of different gender in different time under PTSD, so as to provide scientific suggestions for the placement and the psychological assistance to trauma patients in future. The investigation also provided a scientific basis for the provision of targeted psychological intervention and the development of an overall plan for the psychological intervention. At the same time, based on the psychological assistance station established by the Institute of Psychology of the Chinese Academy of Sciences in the district, the first-hand information of the psychological assistance practice is combined, striving to form the key indicators and technologies for the psychological assessment and intervention of trauma patients, apply these to the combination of biofeedback and sequential psychotherapy and psychological assistance work and to provide the theoretical guidance and effect evaluation scheme for the psychological assistance of patients with trauma.

\section{REFERENCES}

1. Berg BI, Juergens P, Soerensen Y, Savic M, Zeilhofer HF, Schwenzer-Zimmerer K. Traumatology of the facial skeleton in octogenarian patients: aretrospective analysis of 96 cases. $\mathrm{J}$ Craniomaxillofac Surg 2014;42(6):870-3.

2. Aizawa E, Sato Y, Kochiyama T, Saito N, Izumiyama M, Morishita $\mathrm{J}$, et al. Altered cognitive function of prefrontal cortex during error feedback in patients with irritable bowel syndrome, based on fmri and dynamic causal modeling. Gastroenterology 2012;143(5):1188-98.

3. Penson DF. Re: course and predictors of cognitive function in patients with prostate cancer receiving androgen-deprivation therapy: a controlled comparison. J Urol 2016;195(1):97.

4. Naehrig DN, Koh ES, Vogiatzis M, Yanagisawa W, Kwong $\mathrm{C}$, Shepherd HL, et al. Impact of cognitive function on communication in patients with primary or secondary brain tumours. J neurooncol 2015;126(2):299-307.

5. Akhbari B, Salavati M, Ahadi J, Ferdowsi F, Sarmadi A, Keyhani S, et al. Reliability of dynamic balance simultaneously with cognitive performance in patients with acl deficiency and after acl reconstructions and in healthy controls. Knee Surg Sports Traumatol Arthrosc 2015;23(11):3178-85.

6. Trento M, Raballo M, Trevisan M, Sicuro J, Passera P, Cirio $\mathrm{L}$, et al. A cross-sectional survey of depression, anxiety, and cognitive function in patients with type 2 diabetes. Acta Diabetol 2012;49(3):199-203.

7. Jadhav P. A comparison of desflurane and sevoflurane in the recovery of cognitive function after general anesthesia in elderly patients. J Appl Polym Sci 2017;120(3):3278-82.

8. Djurovic M, Pereira AM, Jwa S, Vasovic O, Damjanovic S, Jemuovic $\mathrm{Z}$, et al.Cognitive functioning and quality of life in patients with hashimoto thyroiditis on long-term levothyroxine replacement. Endocrine 2018;1:136-43.

9. Mokhber N, Azarpazhooh A, Orouji E, Rao SM, Khorram B, Sahraian MA, et al. Cognitive dysfunction in patients with 
multiple sclerosis treated with different types of interferon beta: a randomized clinical trial. J Neurol Sci 2014;342(12):16-20.

10. Beatson J, Rao S. Psychotherapy for borderline personality disorder. Br J Psychiatry 2014;186(3):267-8.

11. O'Connor TSJ, Meakes E. Spiritual care and psychotherapy. J Am Ceram Soc 2014;70(1):15-22.

12. Cuijpers P, Huibers M, Ebert DD, Koole SL, Andersson G. How much psychotherapy is needed to treat depression? a metaregression analysis. J Affect Disord 2013;149(1):1-13.

13. Barsaglini A, Sartori G, Benetti S, Pettersson-Yeo W, Mechelli A. The effects of psychotherapy on brain function: a systematic and critical review. Prog Neurobiol 2014;114(1):1-14.

14. Stelingis RA. Contributing to the origins and development of gestalt psychotherapy in lithuania 1993 to 2013. Anal Biochem 2014;361(1):93-101.

15. Yeh CC, Lin F, Hsu CY. A hybrid kmv model, random forests and rough set theory approach for credit rating. KnowledgeBased Sys 2012;33(3):166-72.

This is an open access article distributed under the terms of the Creative Commons Attribution-NonCommercial-ShareAlike 3.0 License, which allows others to remix, tweak, and build upon the work non-commercially, as long as the author is credited and the new creations are licensed under the identical terms

This article was originally published in a special issue: Special issue on "Animal Models \& Experimental Medicine"

Indian J Pharm Sci 2020:82(1)spl issue4;32-29 